\title{
Explore the Lifetime Frontier with MATHUSLA
}

\author{
Cristiano Alpigiani, ${ }^{1, a}$ on behalf of the MATHUSLA Collaboration \\ ${ }^{1}$ University of Washington, Seattle, WA 98195, USA
}

\begin{abstract}
Many extensions of the Standard Model (SM) include particles that are neutral, weakly coupled, and long-lived that can decay to final states containing several hadronic jets. Long-lived particles (LLPs) can be detected as displaced decays from the interaction point, or missing energy if they escape. ATLAS and CMS have performed searches at the LHC and significant exclusion limits have been set in recent years. However, the current searches performed at colliders have limitations. An LLP does not interact with the detector and it is only visible once it decays. Unfortunately, no existing or proposed search strategy will be able to observe the decay of non-hadronic electrically neutral LLPs with masses above $\mathrm{GeV}$ and lifetimes near the limit set by Big Bang Nucleosynthesis $\left(c \tau \sim 10^{7}-10^{8} \mathrm{~m}\right)$. Therefore, ultra-long-lived particles (ULLPs) produced at the LHC will escape the main detector with extremely high probability. MATHUSLA (MAssive Timing Hodoscope for Ultra Stable neutraL pArticles) is a surface detector, which can be implemented with existing technology and in time for the high luminosity LHC upgrade to find such ultra-long-lived particles, whether produced in exotic Higgs decays or more general production modes. The MATHUSLA detector will consist of resistive plate chambers (RPC) and scintillators with a total sensitive area of $200 \times 200 \mathrm{~m}^{2}$. It will be installed on the surface, close to the ATLAS or CMS detectors. A small-scale test detector $\left(\sim 6 \mathrm{~m}^{2}\right)$ is going to be installed on the surface above ATLAS in November 2017. It will consist of three layers of RPCs used for timing/tracking and two layers of scintillators for timing measurements. It will be placed above the ATLAS interaction point to estimate cosmic backgrounds and proton-proton backgrounds coming from ATLAS during nominal LHC operations.
\end{abstract}

\section{The hidden sector}

The discovery of the Higgs boson at the LHC completed the SM, and focused attention on the many central features of our universe that the SM does not address: dark matter (DM), neutrino mass, particle-antiparticle asymmetry (Baryogenesis), hierarchy problem (naturalness). Many BSM (Beyond the Standard Model) theoretical constructs proposed in the past few years that address these phenomena contain long-lived particles with macroscopic decay lengths limited only by Big Bang Nucleosynthesis $(\mathrm{BBN})$ of about $c \tau \lesssim 10^{7}-10^{8} \mathrm{~m}$. Examples include, Supersymmetric (SUSY) models such as mini split SUSY [1], gauge mediation [2], RPV SUSY [3] and Stealth SUSY [4]. Models addressing the hierarchy problem such as Neutral Naturalness [5] and Hidden Valleys [6, 7], and models addressing dark matter [8].

\footnotetext{
ae-mail: Cristiano.Alpigiani@cern.ch
} 


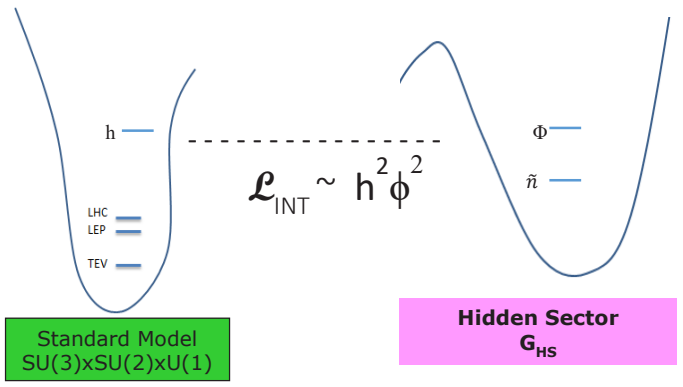

(a)

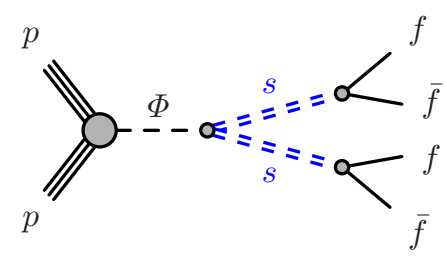

(b)

Figure 1: Coupling between the SM and a hidden sector (a). Feynman diagram for the Higgs decaying into displaced hadronic jets (b).

LLPs occur naturally in coupling to a hidden sector via small portal couplings. For example scalar (Higgs) and vector $\left(\gamma_{d}, Z\right)$ portals $[6,7]$ where the Higgs boson mixes with a hidden sector scalar resulting in a hidden sector Higgs that decays to a pair of hidden sector scalars that carry no SM charge (shown schematically in Figure 1). If the hidden sector scalars are weakly coupled, they will have a long-lifetime and decay far from the IP ( $p p$ interaction point) to a fermion anti-fermion pair (quark anti-quark pairs). The detector signature is a hadronic jet displaced from the IP and no track connecting it to the IP. Similarly the kinetic mixing of a $\gamma_{d}$ results in dark photons that decay to lepton pairs giving rise to a pair of displaced lepton vertices in the detector. The hidden sector particles have no SM quantum numbers and once produced travel through the detector unabated. Once they decay to SM particles a detector signal is observed. The salient signature is a displaced vertex with no tracks connecting to the IP.

Searches for LLPs in the LHC detectors have set significant $c \tau$ exclusion limits from a few centimetres to tens of meters[9, 10]. The large backgrounds from various SM processes (such as QCD jets) limit the $c \tau$ reach of the LHC detectors. The proposed MATHUSLA detector [11], which is a dedicated large volume, air filled detector located on the surface above and somewhat displaced from the ATLAS or CMS IPs, will be sensitive to lifetimes of $10^{7} \mathrm{~m}$ with the expected integrated luminosity of High Luminosity LHC (HL-LHC).

\section{The MATHUSLA detector}

MATHUSLA [11] is a proposed dedicated detector to observe Ultra Long-lived Particles (ULLPs) produced in $p p$ collisions that, because of their long lifetimes, decay to charged SM particles far from the main detector. The HL-LHC will produce $N_{h} \approx 1.5 \times 10^{8}$ Higgs bosons.

When producing ULLPs in exotic Higgs decays, the number of observed ULLP decays is roughly:

$$
N_{\text {obs }} \sim N_{h} \cdot \operatorname{Br}(h \rightarrow \mathrm{ULLP} \rightarrow \mathrm{SM}) \cdot \epsilon_{\text {geometric }} \cdot \frac{L}{b c \tau},
$$

where $L$ is the linear size of the detector along the ULLP's direction of travel, $\epsilon_{\text {geometric }}$ is the chance that the ULLP will pass through the detector (i.e. geometric coverage), and $b$ is the Lorentz boost $|\vec{p}| / m$ of the produced ULLP. Since the Higgs boson is dominantly produced on threshold, if it decays 
to $n$ ULLPs with mass $m_{X}$, their characteristic boost will be

$$
b \sim \frac{m_{h}}{n m_{X}},
$$

so typically $b \lesssim 3$ for $n=2$ and $m_{X} \gtrsim 20 \mathrm{GeV}^{1}$. We focus on $n=2$ which suffices to discuss detector requirements. Assuming the ULLP decays exclusively to the $\mathrm{SM}^{2}$, observation of a few ULLP decays with a lifetime of $c \tau \sim 10^{7} \mathrm{~m}$ requires

$$
L \sim(20 \mathrm{~m})\left(\frac{b}{3}\right)\left(\frac{0.1}{\epsilon_{\text {geometric }}}\right) \frac{0.3}{\operatorname{Br}(h \rightarrow \text { ULLP })} .
$$

ULLPs near the BBN lifetime bound arising from exotic Higgs decays near current limits [12] could be discovered if the detector had a linear size of $\sim 20 \mathrm{~m}$ in the direction of travel and $\sim 10 \%$ geometric coverage and did not have $p p$ collision backgrounds.

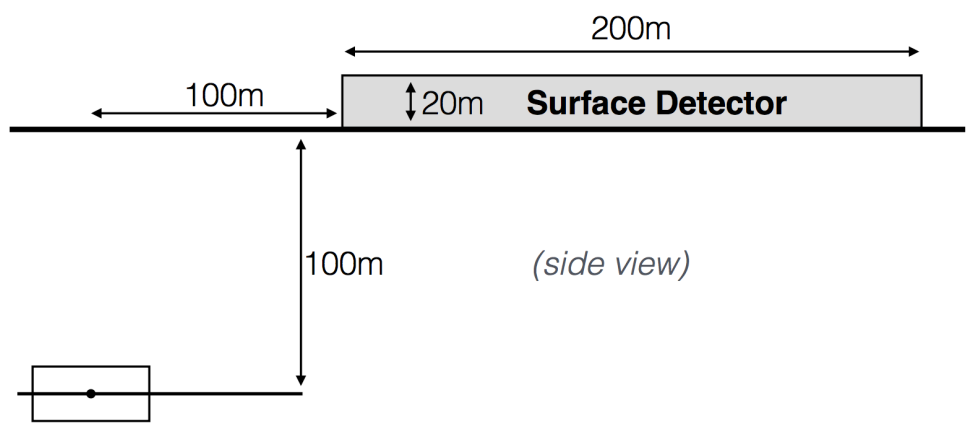

Figure 2: Possible geometric configurations for the MATHUSLA surface detector at the HL-LHC. Grey shading indicates areas assumed to be sensitive to LLP decays. The surface detector is a $200 \mathrm{~m}$ square building, centred along the beam line.

Placing a sufficiently large area detector on the surface, as shown in Figure 2, above the ATLAS or CMS collision points ( 80 to $100 \mathrm{~m}$ below) gives sufficient acceptance to reach the BBN lifetime limit [11]. The 80 to 100 meters of rock removes most backgrounds associated with $p p$ collisions, but a large background of cosmic muons and backgrounds from high energy muons and neutrinos coming from the IP must be rejected. The proposed detector concept, shown in Figure 2, is basically a large box of about $200 \times 200 \mathrm{~m}^{2}$ surrounded by a layer of scintillators, a robust tracking system (about 5 layers of tracking chambers) and a few meter air decay volume. Figure 3 (a) shows two possible displaced vertex signals from LLP decays, while potential backgrounds are shown symbolically in Figure 3 (b). They include a cosmic ray muon rate of about $10^{6} \mathrm{~Hz}$. The about $20 \mathrm{~m}$ height results in a $\Delta \mathrm{t}=70 \mathrm{~ns}$ top to bottom and scintillators have $1.5 \mathrm{~ns}$ or better time resolution.

Scintillators provide good time resolution and RPCs are capable of both good space and time resolution needed for vertex reconstruction and cosmic ray rejection. Having tracking chambers that provide both coordinate and time of hits is a powerful handle for rejection backgrounds. The expected

\footnotetext{
${ }^{1} n>2$ typically implies higher-dimensional operators, cascade decays in the hidden sector, or a hidden confining gauge group that produces a shower.

${ }^{2}$ Naively, a coincidence of small widths is required for a ULLP to decay to both SM and hidden sector particles.
} 


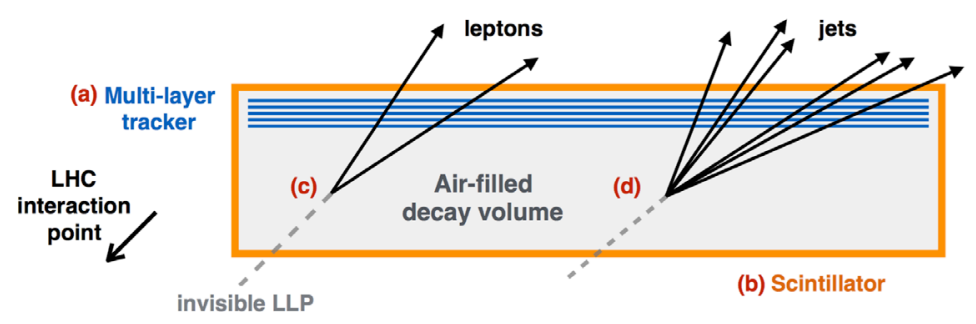

(a)

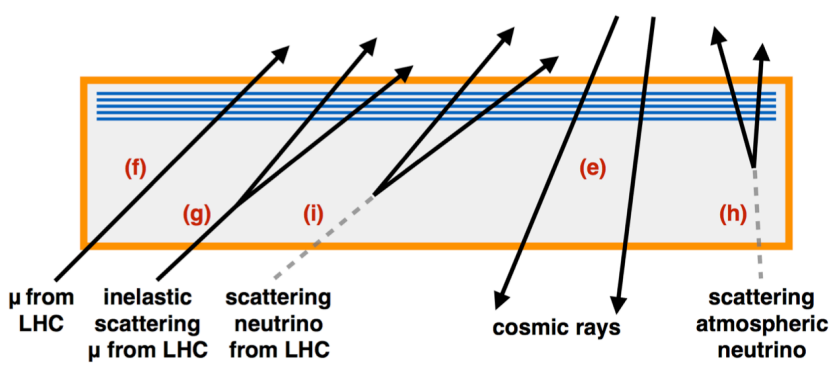

(b)

Figure 3: Schematic of a possible design for MATHUSLA, with a robust multi-layer tracker at the top and a segmented scintillator veto surrounding the entire detector. Also shown are two possible displaced vertex signals from LLP decays (a) and the five most important backgrounds (b). Black arrows indicate charged particles and their direction of travel.

rate of LHC collision muons is about $10 \mathrm{~Hz}$ and scintillator and are rejected by timing and entrance hit position. Upward going atmospheric neutrinos are estimated to be of order 10 to 100 per year and most can be rejected using time of flight. In addition, such non-collision backgrounds can be measured when there are no LHC collisions. Neutrinos from LHC collisions are estimated to a few events during the entire HL-LHC data taking period. This needs more work but appears to be a subdominant background.

\section{The MATHUSLA test module}

Several efforts are underway to develop simulations of the backgrounds expected in MATHUSLA. For muons and neutrinos traveling upwards, the idea is to create a "gun" that shoots particles into MATHUSLA, while for cosmic muons the plan is to use the standard cosmic ray simulations. Nevertheless, the simulations need to be validated (and tuned) using real data. For this reason a test detector is fundamental.

Figure 4 shows the basic design of the test module. It is a much simpler version of the final detector, but sufficient for our current goals and fundamental to provide information for the design of the main detector. Top and bottom scintillator layers are coming from Tevatron DØ forward muon trigger scintillators, while the RPCs (provided by University of Tor Vergata, Rome) are coming from the cosmic ray experiment Argo in Tibet.

The test detector will be installed in the surface zone above the ATLAS detector in November 2017 and it will allow to perform background tests with different LHC operating conditions. 


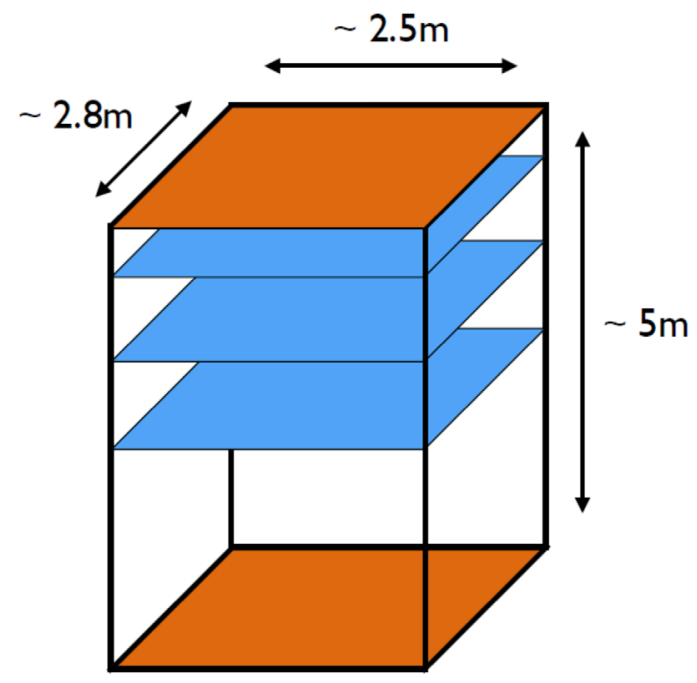

Figure 4: Test module design. Top and bottom are scintillator layers from Tevatron DØ (forward muon trigger scintillators), between these two 3 layers of RPCs are installed. Twelve RPC chambers $\left(1.25 \times 2.8 \mathrm{~m}^{2}\right)$ are used to form $3 \mathrm{RPC}$ planes (4 RPC chambers per plane).

\section{MATHUSLA and cosmic rays}

The combination of a large area detector of atmospheric showers, that can measure both electron and muon particles, with a LHC detector, that can measure only muons, provides a more complete picture of cosmic air showers. In this framework, MATHUSLA can allow more detailed studies of the core structure, crucial to determine the atomic number of the primary cosmic particle. 


\section{References}

[1] G.F. Giudice, A. Romanino, Split Supersymmetry, Nucl. Phys. B 699 (2004) 65-89, 10.1016/j.nuclphysb.2004.11.048, [arXiv: hep-ph/0406088].

[2] G. F. Giudice, R. Rattazzi, Theories with Gauge-Mediated Supersymmetry Breaking, Phys. Rept. 322 (1999) 419-499, 10.1016/S0370-1573(99)00042-3, [arXiv: hep-ph/9801271].

[3] R. Barbier et al., R-parity violating supersymmetry, Phys. Rept. 420 (2005) 1-202, 10.1016/j.physrep.2005.08.006, [arXiv: hep-ph/0406039].

[4] J. Fan, M. Reece, J. T. Ruderman, Stealth Supersymmetry, JHEP 11 (2011) 12, 10.1007/JHEP11(2011)012, [arXiv: 1105.5135 [hep-ph]].

[5] D. Curtin, C. B. Verhaaren, Discovering Uncolored Naturalness in Exotic Higgs Decays, JHEP 12 (2015) 72, 10.1007/JHEP12(2015)072, [arXiv: 1506.06141 [hep-ph]].

[6] M. J. Strassler, K. M. Zurek, Echoes of a Hidden Valley at Hadron Colliders, Phys. Lett. B 651 (2007) 374-379, 10.1016/j.physletb.2007.06.055, [arXiv: hep-ph/0604261].

[7] M. J. Strassler, Possible Effects of a Hidden Valley on Supersymmetric Phenomenology, [arXiv:hep-ph/0607160].

[8] D. Tucker-Smith, N. Weiner, Inelastic dark matter, Phys. Rev. D 64 (2001) 043502, 10.1103/PhysRevD.64.043502, [arXiv: hep-ph/0101138].

[9] ATLAS Collaboration, Search for long-lived, weakly-interacting particles that decay to displaced hadronic jets in proton-proton collisions at $\sqrt{s}=8 \mathrm{TeV}$ with the ATLAS detector, Phys. Rev. D 92 (2015) 012010, 10.1103/PhysRevD.92.012010, [arXiv: 1504.03634 [hep-ex]].

[10] ATLAS Collaboration, Search for heavy long-lived multi-charged particles in pp collisions at $\sqrt{s}=8 \mathrm{TeV}$ using the ATLAS detector, Eur. Phys. J. C 75 (2015) 362, 10.1140/epjc/s10052-0153534-2, [arXiv: 1504.04188 [hep-ex]].

[11] J. P. Chou, D. Curtin, H. J. Lubatti, New detectors to explore the lifetime frontier, Phys. Lett. B, 767 (2017) 29-36, 10.1016/j.physletb.2017.01.043, [arXiv: 1606.06298 [hep-ph]].

[12] CMS Collaboration, Searches for invisible decays of the Higgs boson in pp collisions at $\sqrt{s}=7,8$, and $13 \mathrm{TeV}$, JHEP 02 (2017) 135, 10.1007/JHEP02(2017)135, [arXiv: 1610.09218 [hep-ex]].

[13] C. Csaki, M. Geller, O. Telem, A. Weiler, The Flavor of the Composite Twin Higgs, JHEP 09 (2016) 146, 10.1007/JHEP09(2016)146, [arXiv: 1512.03427 [hep-ph]].

[14] CMS Collaboration, CMS physics: Technical design report, CERN-LHCC-2006-001, CMSTDR-008-1.

[15] D. A. Timashkov, A. A. Petrukhin, New results on muon inelastic cross section and energy loss in rock, Proceedings 29th ICRC Pune 2005 Vol. 9 p. 89, [arXiv: hep-ph/0611008]. 